\title{
Downregulation of microRNA-210 inhibits osteosarcoma growth in vitro and in vivo
}

\author{
CHANGJIAN LIU* and XIN TANG* \\ Department of Orthopaedics, The First Affiliated Hospital of Dalian Medical University, Dalian, Liaoning 116011, P.R. China
}

Received August 5, 2014; Accepted April 16, 2015

DOI: $10.3892 / \mathrm{mmr} .2015 .3880$

\begin{abstract}
MicroRNA-210 (miR-210), the master hypoxamir, has various roles in the development of certain cancer types. It has been reported that miR-210 expression was upregulated in patients with osteosarcoma (OS). However, little is known regarding its role in the development of human OS. In the present study, to explore the feasibility of miR-210 as an effective therapeutic target, miR-210 inhibitor was transfected into the osteosarcoma cell line MG-63 cells, and cell proliferation, colony formation, cycle, apoptosis, migration and invasion were assessed. It was found that miR-210 downregulation significantly suppressed clonogenicity, migration and invasion, as well as induced cell apoptosis, increased the percentage of cells in G1 phrase and decreased the percentage of cells in S phase in vitro. In addition, the effect of miR-210 on tumor growth was evaluated in vivo. The results indicated that miR-210 downregulation significantly suppressed tumor growth in nude mouse models. In conclusion, the findings of the present study suggested that miR-210 is a potential therapeutic agent for the treatment of OS.
\end{abstract}

\section{Introduction}

Osteosarcoma (OS) is the most common primary bone malignancy in children and young adults $(1,2)$. In spite of extensive advancements in diagnostic methods and surgical techniques in recent years, the five-year survival rate of osteosarcoma patients remains poor at $25-40 \%$ due to the high frequency of pulmonary metastasis (3). Therefore, an improved understanding of the mechanisms underlying osteosarcoma development and progression is urgently required to optimize therapeutic options or develop novel effective therapies.

Correspondence to: Professor Changjian Liu or Dr Xin Tang, Department of Orthopaedics, The First Affiliated Hospital of Dalian Medical University, 222 Zhongshan Road, Dalian, Liaoning 116011, P.R. China

E-mail: liuchangjian8514@126.com

E-mail: tangxin851@163.com

${ }^{*}$ Contributed equally

Key words: osteosarcoma, microRNAs, microRNA-210, tumor growth
MicroRNAs (miRNAs or miRs) are a class of highly conserved short non-coding small RNAs, usually 18-25 nucleotides in length, which inhibit translation and cleave mRNA by base-pairing to the $3^{\prime}$ untranslated region of the target genes (4-8). In mammals, miRNAs were predicted to regulate the activity of $>50 \%$ of all protein-coding genes (9). Due to their widespread regulating functions, miRNAs are able to regulate a variety of biological processes, including cell proliferation, differentiation, migration, metabolism and apoptosis (10). For cancers, homeostatic expression of miRNAs is disrupted, which results in aberrant gene expression in tumor initiation, development and metastasis. An increasing number of studies has pointed out that multiple miRNAs are involved in carcinogenesis and tumor progression in OS (11-15). For example, miR-124 was reported to function as a tumor oncogene by downregulating putative tumor suppressor 1 (11). miR-145 exerts tumor-suppressive effects in osteosarcoma genesis through suppression of Rho-associated, coiled-coil containing protein kinase 1 (12). A recent miRNA microarray analysis by Lulla et al (13) identified 22 differentially expressed miRNAs in osteosarcomas and found that miR-210, a key factor in the hypoxic response, was significantly elevated in osteosarcoma tissues compared with that in the corresponding controls. Subsequently, Cai et al (14) further confirmed this result, and found that miR-210 expression was significantly increased in osteosarcoma tissues compared with that in corresponding non-cancerous bone tissues, and that miR-210 upregulation more frequently occurred in osteosarcoma tissues with large tumor size, poor response to pre-operative chemotherapy and positive metastasis. In addition, miR-210 has been demonstrated to be upregulated in all cell types under hypoxic conditions (15). However, the precise role of miR-210 in OS cells remains elusive at present.

Therefore, the aim of the present study was to identify the detailed roles of miR-210 in OS cells in vitro and in vivo in order to determine its utility in OS therapy. The effect of downregulation of miR-210 on clonogenicity, migration and invasion, as well as apoptosis and cell cycle were examined in vitro. In addition, the effect of miR-210 on tumor growth in nude mice was assessed. The findings of the present study implied that miR-210 is a potential therapeutic target for OS.

\section{Materials and methods}

Cell lines and cell culture. The human osteosarcoma cell lines MG63, Saos-2, U2OS and the human osteoblast cell line h-OB 
were obtained from the American Type Culture Collection (ATCC, Manassas, VA, USA). Cells were grown in Dulbecco's modified Eagle's medium (DMEM; HyClone, GE Healthcare, Little Chalfont, UK) supplemented with $10 \%$ fetal bovine serum (FBS; Gibco-BRL, Invitrogen Life Technologies, Carlsbad, CA, USA), 2 mM glutamine, $100 \mathrm{U} / \mathrm{ml}$ penicillin and $100 \mathrm{mg} / \mathrm{ml}$ streptomycin (Sigma-Aldrich, St Louis, MO, USA) at $37^{\circ} \mathrm{C}$ in a $5 \% \mathrm{CO}_{2}$ atmosphere.

miRNA transfection. miR-210 inhibitor (5'-TCAGCCGCT GTCACACGCACAG-3') or negative control miRNA (5'-GTG TAACACGTCTATACGCCCA-3') (Applied Biosystems, Thermo Fisher Scientific, Waltham, MA, USA) were transiently transfected into MG63 cells in six-well plates using Lipofectamine 2000 (Invitrogen Life Technologies) according to the manufacturer's instructions at a concentration of $100 \mathrm{nM}$.

Reverse transcription quantitative polymerase chain reaction (RT-qPCR). Transfected cells were harvested by centrifugation at $1,000 \times \mathrm{g}$ for $5 \mathrm{~min}$ at room temperature after $48 \mathrm{~h}$, and total RNA enriched with small RNAs was isolated using the mirVana miRNA Isolation kit (Ambion, Life Technologies, Thermo Fisher Scientific) according to manufacturer's instructions. Concentration and purity of RNA were determined using a GeneQuant RNA/DNA Calculator (GE Healthcare Life Sciences, Uppsala, Sweden), and miRNA was reversely transcribed using the One Step Prime script miRNA cDNA Synthesis kit (Qiagen, Hilden, Germany) and then quantified by real-time RT-PCR using SYBR Premix Ex Taq (Takara, Dalian, China). All PCR reactions were monitored using an ABI 7900 Fast system (Applied Biosystems). The primers of miR-210 (CUGUGCGUGUGACAGCGGCUGA) and RNU6B (CGCAAGGAUGACACGCAAAUUCGUGAA GCGUUCCAUAUUUUU) were purchased from Applied Biosystems. The expression levels of RNU6B were used as an internal control. PCR was performed at $95^{\circ} \mathrm{C}$ for $10 \mathrm{~min}$, followed by 40 cycles of $95^{\circ} \mathrm{C}$ for $15 \mathrm{sec}$ and $60^{\circ} \mathrm{C}$ for $1 \mathrm{~min}$. The relative quantified miRNA levels were calculated as the fold change after normalization to U6 RNA using the equation $2^{-\Delta \Delta \mathrm{Ct}}$ with the Rotor-Gene 6000 Series Software 1.7 (Qiagen, Hilden, Germany). miR-210 levels in the negative control cells were compared with those of untransfected cells and non-significant changes $<5 \%$ were observed. All experiments were repeated three times to reduce curve-derived variance.

Cell prolifration and colony formation assay. Cell viability was examined using an MTT assay. In brief, MG63 cells were seeded into 96-well plates at a density of 2,000 cells/well containing $100 \mu \mathrm{l}$ culture medium and cultured overnight, and cells were then transfected with $100 \mathrm{nM}$ miR-210 inhibitor or negative control. Non-transfected cells (blank) were used as a control. Fresh complete medium was changed for cells at $24 \mathrm{~h}$ post-transfection except for those to be tested for viability at this time-point. Every 24-h interval, $20 \mu 15 \mathrm{mg} / \mathrm{ml}$ MTT (Sigma-Aldrich) was added into each corresponding test well and incubated for $4 \mathrm{~h}$ in a humidified incubator. The supernatant was then discarded and $200 \mu \mathrm{l}$ dimethylsulfoxide (DMSO; Sigma-Aldrich) was added to each well to dissolve the formazan. The optical density (OD) was evaluated by measuring the absorbance at a test wavelength of $490 \mathrm{~nm}$ and a reference wavelength of $630 \mathrm{~nm}$ using a SpectraMax M3 ELISA spectrophotometer (Molecular Devices, LLC, Sunnyvale, CA, USA). Wells without cells (DMSO alone) were used as a control to normalize the results. Each test was performed daily for five consecutive days and repeated in eight wells. The experiments were repeated three times independently.

For the colony formation assay, cells were seeded in six-well plates at a low density $\left(1 \times 10^{3}\right.$ cells/well) and cultured overnight. Cells were then transfected with $100 \mathrm{nM}$ miR-210 inhibitor or negative control and cultured for 7 days. Cells were then fixed with $4 \%$ paraformaldehyde (Sigma-Aldrich) for $20 \mathrm{~min}$ and counted after staining with $1 \%$ crystal violet (Sigma-Aldrich). The experiments were performed in triplicate wells for at least three times.

Cell cycle analysis by flow cytometry. $24 \mathrm{~h}$ prior to transfection, MG63 cells were seeded into six-well plates at a density of $1 \times 10^{5}$ cells/well. Cells were then transfected with $100 \mathrm{nM}$ miR-210 inhibitor or corresponding negative control. $48 \mathrm{~h}$ after transfection, the transfected MG63 cells in the logarythmic growth phase were stained with the DNA-binding dye propidium iodide (PI; $50 \mathrm{mg} / \mathrm{ml}$; Sigma-Aldrich) and RNase $\left(1.0 \mathrm{mg} / \mathrm{ml}\right.$; Sigma-Aldrich) for $30 \mathrm{~min}$ at $37^{\circ} \mathrm{C}$ in the dark and examined by fluorescence-activated cell-sorting (FACS) using a flow cytometer (FACScan; BD Biosciences, Franklin Lakes, NJ, USA), and DNA histograms were analyzed with CellQuest v 3.3 software (BD Biosciences).

Cell apoptosis assay. MG63 cells were seeded into six-well plates at a density of $1 \times 10^{5}$ cells/well and cultured overnight. Then, cells were transfected with $100 \mathrm{nM}$ miR-210 inhibitor or corresponding negative control. $48 \mathrm{~h}$ after transfection, MG63 cells were incubated with Annexin V/PI (Sigma-Aldrich) for $15 \mathrm{~min}$ at room temperature in the dark. The cells were then analyzed by flow cytometry with fluorescence-activated cell-sorting (FACS) flow cytometer (FACScan; BD Biosciences). Each test was repeated in triplicate. Experiments were performed in triplicate.

In addition, caspase- 3 , caspase- 8 and caspase- 9 activity were tested using Caspase Colorimetric Protease Assay kits (EMD Millipore, Billerica, MA, USA) as an additional indicator of apoptosis.

Caspase activity. The activity of caspase- 3 , caspase- 8 and caspase- 9 was determined by caspase colorimetric protease assay kits (EMD Millipore) according to the manufacturer's instructions. In brief, MG63 cells were seeded into six-well plates at a density of $1 \times 10^{5}$ cells/well and cultured overnight. Cells were then transfected with $100 \mathrm{nM}$ miR-210 inhibitor or corresponding negative control and cultured for $48 \mathrm{~h}$. The cells were washed twice with ice-cold phosphate-buffered saline (PBS; Sigma-Aldrich) and harvested by centrifugation at $800 \mathrm{xg}$ for $5 \mathrm{~min}$. The cell pellets were then lysed in $150 \mu \mathrm{l}$ buffer provided in the kit. Protein concentrations of lysates were measured by the Lowry method. An aliquot of lysates (80 $\mu \mathrm{l})$ was incubated with $10 \mu \mathrm{l}$ substrate of each caspase at $37^{\circ} \mathrm{C}$ for $1 \mathrm{~h}$. Samples were analyzed using a Multiskan Spectrum microplate reader (Thermo Fisher Scientific) at 
$405 \mathrm{~nm}$. The relative caspase activity of the negative control group was referred to as 100 .

Wound-healing assays. MG63 cells were treated with miR-210 inhibitor or corresponding negative control when cells were grown to $80-90 \%$ confluence in 24 -well plates. At $24 \mathrm{~h}$ following transfection, linear scratch wounds were created on the confluent cell monolayers with a $100-\mu 1$ pipette tip. To stop cells from entering the cell cycle prior to wounding, cells were maintained in serum-free medium. To visualize migrating cells and wound healing, images were captured at 0 and $24 \mathrm{~h}$ using an IX51 inverted microscope (Olympus, Tokyo, Japan). Five random fields of view were selected from each well and the cells in three wells of each group were determined.

Cell invasion assay. For the cell invasion assay, cells transfected with miR-210 inhibitor or corresponding negative control were harvested and re-suspended in serum-free DMEM. Aliquots of cells $\left(5 \times 10^{4}\right)$ were placed in chambers coated with $150 \mathrm{mg}$ Matrigel (BD Biosciences), which were fitted into 24-well plates and incubated for $24 \mathrm{~h}$ in DMEM with $10 \%$ FBS. Cells remaining on the upper surface of the membranes were removed, whereas those adhering to the lower surface were fixed with ice-cold methanol (Sigma-Aldrich) and stained with $1 \%$ crystal violet. Stained cells were visualized and counted under the IX51 inverted microscope (Olympus). Experiments were performed in triplicate.

Western blot analysis. At $48 \mathrm{~h}$ post-transfection, MG63 cells were washed with pre-chilled PBS and then subjected to lysis in a radioimmunoprecipitation assay buffer containing $0.5 \%$ SDS and 3\% proteinase inhibitor cocktail (Sigma-Aldrich) on ice for $30 \mathrm{~min}$. The lysate was then centrifuged at $14,000 \mathrm{xg}$ for $20 \mathrm{~min}$, and the supernatant was harvested as total proteins for subsequent analysis. The total concentration of protein was measured using a bicinchoninic acid protein assay kit (Boster, Beijing, China). Equal amounts of protein lysates $(20 \mu \mathrm{g}$ per lane) was separated by $8-15 \%$ SDS-PAGE (Sigma-Aldrich) and then electrotransferred onto nitrocellulose membranes (Invitrogen Life Technologies). The membranes were blocked with Tris-buffered saline containing Tween 20 (TBST; Sigma-Aldrich) with 5\% non-fat milk powder for $2 \mathrm{~h}$ and incubated with the following primary antibodies: Rabbit monoclonal anti-human anti-matrix metalloproteinase (MMP)-2 (\#13132; 1:1,000; Cell Signaling Technology, Danvers, MA, USA); mouse monoclonal anti-human anti-MMP-9 (sc-12759; 1:2,000; Santa Cruz Biotechnology, Inc., Dallas, TX, USA); rabbit polyclonal anti-human anti-vascular endothelial growth factor (VEGF; \#2445; 1:2,000; Cell Signaling Technology) and mouse monoclonal anti-human anti- $\beta$-actin (sc-47778; 1:10,000; Santa Cruz Biotechnology) overnight at $4^{\circ} \mathrm{C}$. After three washes with TBST, the membranes were incubated with polyclonal goat anti-mouse (sc-2005) or goat anti-rabbit (sc-2004) horseradish peroxidase-conjugated secondary antibodies (1:10,000; Santa Cruz Biotechnology, Inc.) for $2 \mathrm{~h}$ at room temperature. Blots were washed three times with TBST again, and proteins were detected by enhanced chemiluminescence (ECL; ECL-Plus; Thermo Fisher Scientific). $\beta$-actin was used as a loading control. The optical density of protein
A

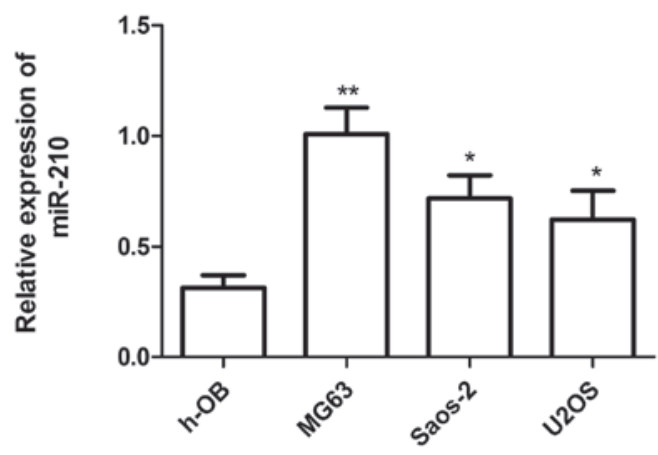

B

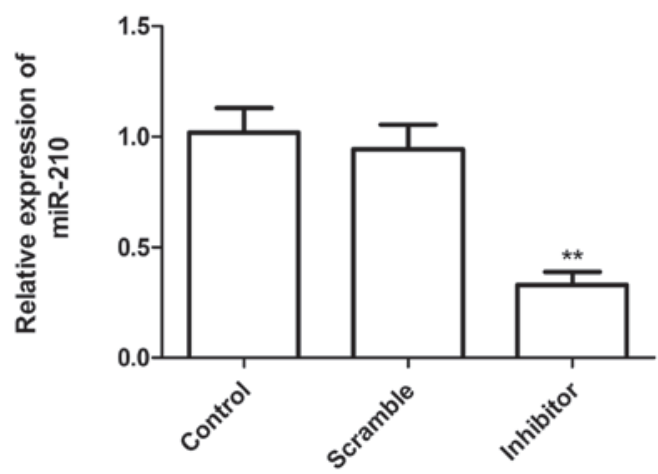

Figure 1. miR-210 expression and miR-210 inhibitor transfection in osteosarcoma cells. (A) Expression of miR-210 analyzed by reverse transcription quantitative polymerase chain reaction and normalized to RNU6B in three osteosarcoma cell lines. The expression of miR-210 in h-OB osteoblasts was used as a control, ${ }^{* *} \mathrm{P}<0.01$ vs. control. (B) miR-210 expression levels were assayed in miR-210 inhibitor-transfected MG63 cells. RNU6B was used as an internal loading control to normalize the results. ${ }^{* *} \mathrm{P}<0.01$, vs. blank control. All values are expressed as the mean \pm standard deviation. $\mathrm{miR}$, microRNA.

fragments was quantified by Quantity One v.4.6.1 software (Bio-Rad, Billerica, MA, USA).

Tumor growth in vivo. Logarithmically growing MG63 cells transfected with miR-210 inhibitor or negative control were harvested and re-suspended in PBS, and aliquots of $2 \times 10^{6}$ cells inoculated subcutaneously into five-week-old male BALB/c nude mice (HFK Bio-Technology, Co., Ltd; Beijing, China), respectively. The mice ( $\mathrm{n}=10$ per group) were maintained in specific pathogen-free conditions with free access to food and water, and were maintained at normal $12 \mathrm{~h} \mathrm{light/dark} \mathrm{cycles.} \mathrm{After} 30$ days, mice were sacrificed by cervical dislocation, followed by surgical excision of tumors. Tumors were weighed and their volumes were calculated as $0.5236 \mathrm{x}$ width ${ }^{2} \mathrm{x}$ length. All animal experiments were performed following the standards of animal care as outlined in the Guide for the Care and Use of Experimental Animals of Dalian Medical University Jilin University (Dalian, China), following a protocol approved by the Ethics Committees of the Disease Model Research Center, Dalian Medical University (Dalian, China).

Statistical analysis. All values are expressed as the mean \pm standard deviation. Student's $t$ test and one-way analysis of variance were used to determine statistical significance. All statistical tests were two-sided. $\mathrm{P}<0.05$ was 
A

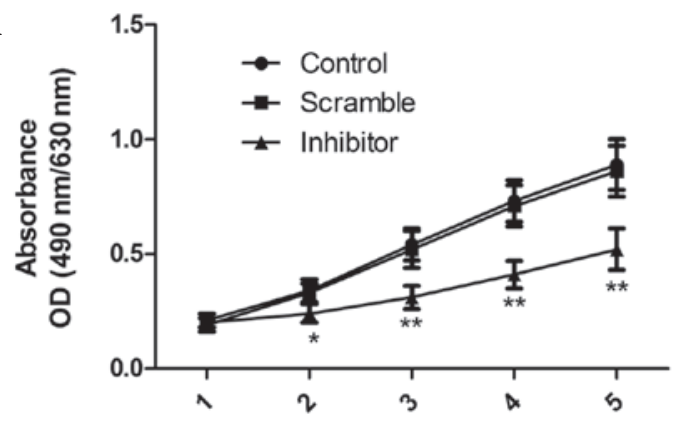

B

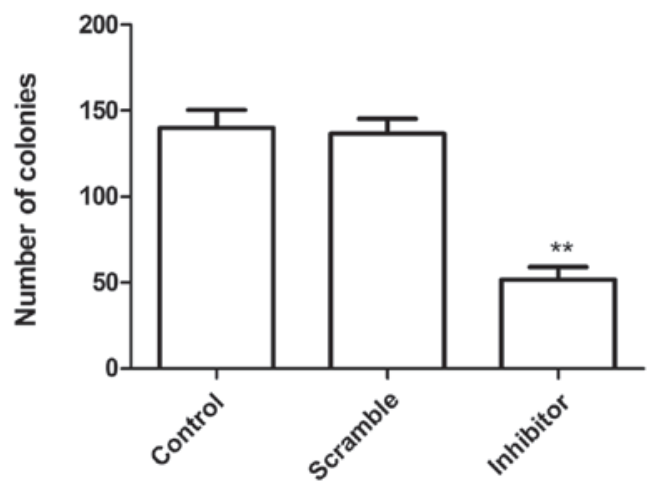

Figure 2. miR-210 inhibitor suppresses cell proliferation and cell colony formation of MG63 cells. (A) MTT assay was performed to analyze the effect of miR-210 inhibitor on cell proliferation of MG63 cells. (B) The effects of miR-210 inhibitor on MG63 cell colony formation were determined. " $\mathrm{P}<0.05$; ${ }^{* *} \mathrm{P}<0.01$, vs. blank control. All values are expressed as the mean \pm standard deviation. OD, optical density; miRNA, microRNA.

A

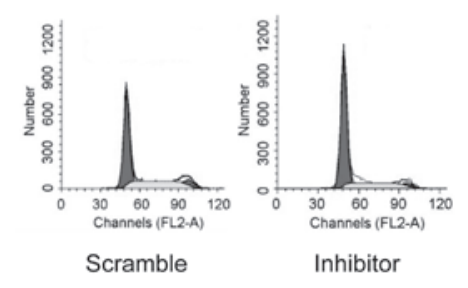

D

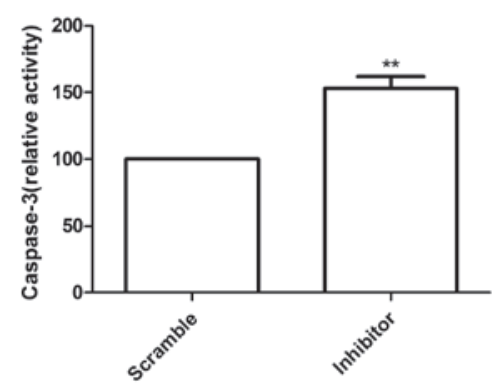

B

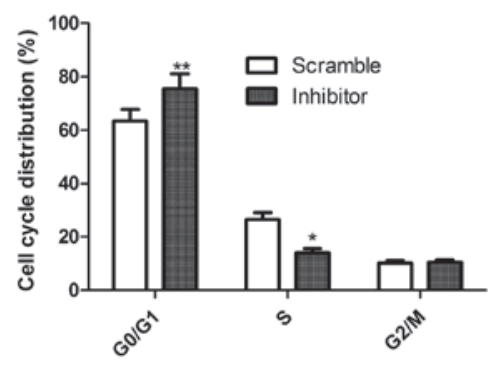

$\mathbf{E}$

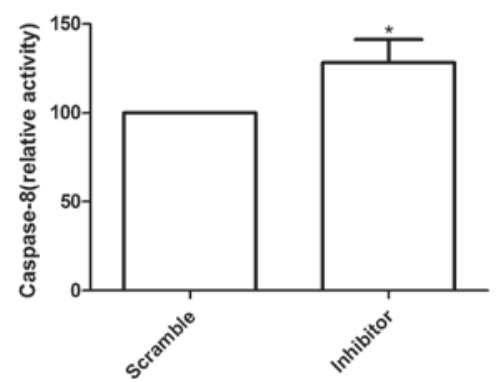

$\mathbf{C}$
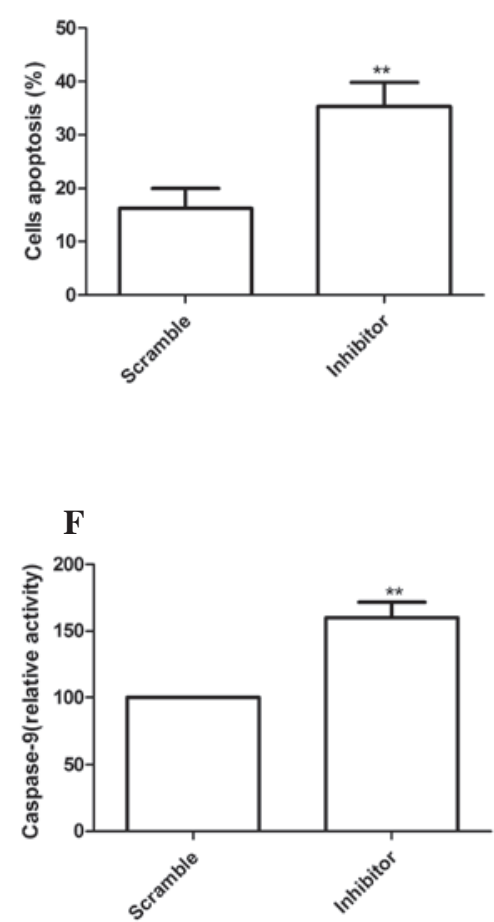

Figure 3. MicroRNA-210 inhibitor induces cell cycle block and cell apoptosis in SKVO3 cells. (A) Cell cycle distribution. (B) Percentages of cells in each phase of the cell cycle. (C) Apoptosis was determined by flow cytometry. (D-F) Caspase-3, caspase-8 and caspase-9 activity were determined by ELISA. "P<0.05; ${ }^{* *} \mathrm{P}<0.01$, vs. the scramble group. All values are expressed as the mean \pm standard deviation.

considered to indicate a statistically significant difference between values. Statistical analyses were performed using SPSS 16.0 statistics software (SPSS Inc, Chicago, IL, USA) and GraphPad Prism version 5.01 (GraphPad Software, La Jolla, CA, USA) for Windows ${ }^{\circledR}$.

\section{Results}

miR-210 is upregulated in human osteosarcoma cell lines. To elucidate the role of miR-210 in human osteosarcoma, the expression levels of miR-210 in three human osteosarcoma cell lines, U2OS, Saos-2 and MG63, as well as in the human osteoblast cell line h-OB were determined using RT-qPCR.
The h-OB cell line was used as a control. The results showed that osteosarcoma cell lines (U2OS, Saos-2 and MG-63) exhibited higher miR-210 expression as compared with that in the human osteoblast cell line (h-OB) (Fig. 1A). These results indicated that miR-210 is significantly upregulated in osteosarcoma cell lines.

To investigate the function of miR-210, miR-210 inhibitor or corresponding scrambled control were transfected into MG63 cells (which had the highest expression levels of miR-210). At $24 \mathrm{~h}$ after transfection, miR-210 expression was evaluated by RT-qPCR. The results showed that miR-210 significantly decreased the expression of miR-210 compared with that in the scrambled group and the blank group. In addition, no statisti- 
A

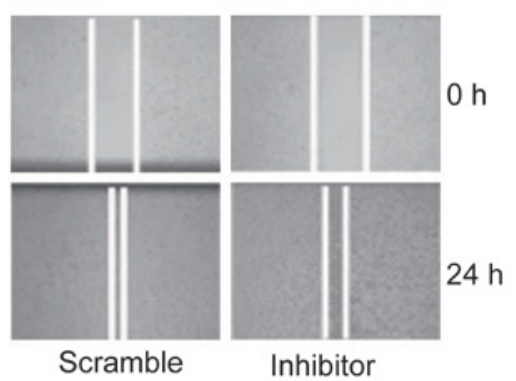

D

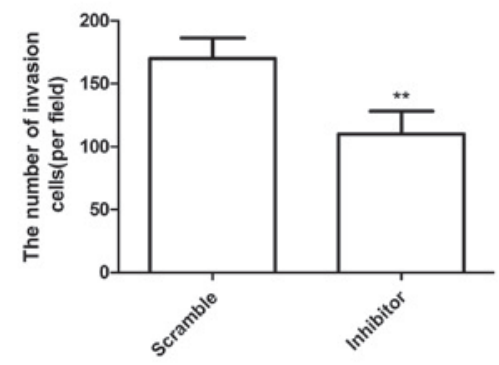

B

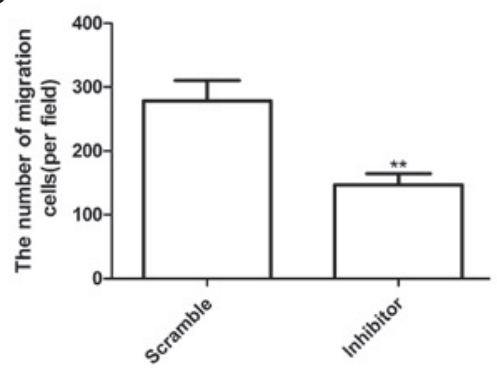

E
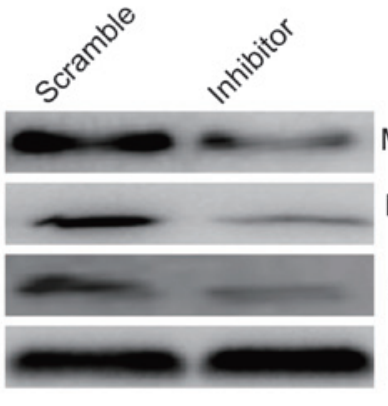

C
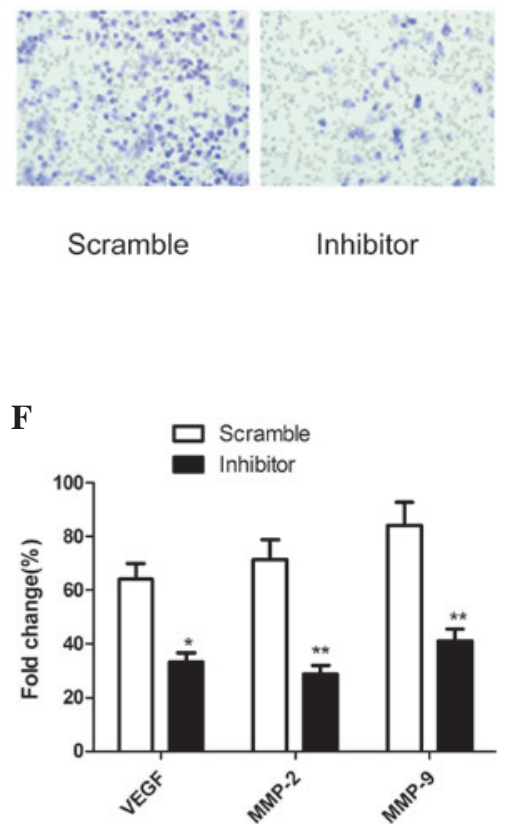

Figure 4. miR-210 inhibitor reduced cell migration and invasion of MG63 cells. (A) Cell migration was determined using the wound-healing assay, magnification, x200. (B) Quantified numbers of migrated cells. (C) Cell invasion was determined by a Matrigel Transwell assay, magnification, x200. (D) Quantified number of invaded cells. (E) Western blot analysis of VEGF, MMP-2 and MMP-9 protein expression after transfection with miR-210 inhibitor and corresponding scrambled control. $\beta$-actin was used as an internal control. (F) Relative quantification of VEGF, MMP-2 and MMP-9 protein by densitometric analysis. ${ }^{*} \mathrm{P}<0.05 ;{ }^{* *} \mathrm{P}<0.01$, vs. the negative control. All values are expressed as the mean \pm standard deviation. VEGF, vascular endothelial growth factor; MMP, matrix metalloproteinase; miR, microRNA.

A
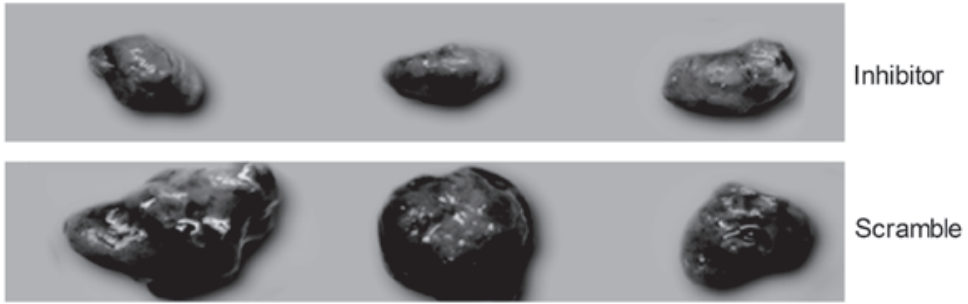

B

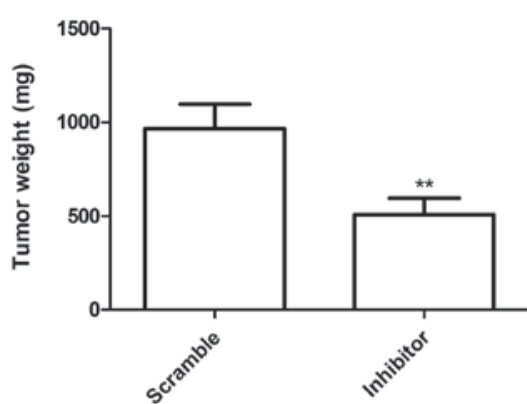

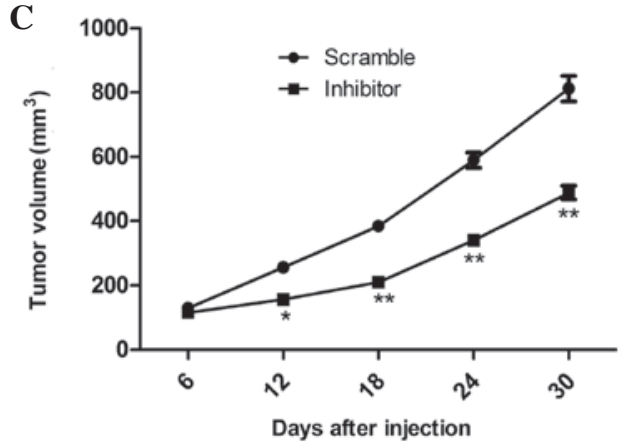

Figure 5. MicroRNA-210 inhibitor suppressed tumor growth in nude mice. (A) Images of tumor tissue from various treatment groups collected after sacrification at day 30. (B) Tumor wet weights measured after sacrification at day 30. (C) Tumor volumes were measured on the indicated days. " $\mathrm{P}<0.05$; ${ }^{* * *} \mathrm{P}<0.01$, vs. the scrambled group. All values are expressed as the mean \pm standard deviation.

cally significant difference in miR-210 expression was found between the scrambled and blank control groups (Fig. 1B).

miR-210 inhibition suppresses cell proliferation and colony formation of MG63 cells. The proliferation rates of MG63 cells were determined via MTT assay following transfec- tion with miR-210 inhibitor. The cell proliferation curve is depicted in Fig. 2A. The results demonstrated that the viability of MG63 cells was markedly decreased by transfection with miR-210 inhibitor compared with that in the scrambled and the blank group, and the suppressive effect of miR-210 inhibitor on cell proliferation was observed from 
day 2 onwards, becoming more obvious on days 4 and 5 $(\mathrm{P}<0.05$; Fig. 2A). No statistically significant difference was observed between the proliferation rate of the blank and that of the scrambled group (Fig. 2A).

In addition, the present study determined the effect of miR-210 on cell colony formation of MG63 cells. The results showed that miR-210 inhibitor significantly inhibited cell colony formation compared to that in the blank and scrambled group $(\mathrm{P}<0.05$; Fig. $2 \mathrm{~B})$. No statistically significant difference was observed rate between the proliferation rate in the blank and that of the scrambled group. These findings suggested that the downregulation miR-210 greatly inhibited cell proliferation and colony formation in MG63 cells.

miR-210 inhibition induces G1 arrest and cell apoptosis in MG63 cells. To determine the effects of miR-210 on the cell cycle, FACScan flow cytometry assays were performed. The results demonstrated that miR-210 inhibitor was able to increase the percentage of cells in G1 phase, while decreasing the percentage of cells in $\mathrm{S}$ phase compared with that in the scramble group $(\mathrm{P}<0.05$, Fig. $3 \mathrm{~A}$ and $\mathrm{B})$.

Next, the role of miR-210 in MG63-cell apoptosis was assessed using Annexin V/PI staining and flow cytometric analysis. The results showed that the miR-210 inhibitor significantly induced cell apoptosis compared with that in the scrambled group $(\mathrm{P}<0.01$; Fig. $3 \mathrm{C})$.

Finally, the effects of miR-210 on caspase-3, caspase- 8 and caspase- 9 activity were assessed using ELISA. As shown in Fig. 3D-F, caspase-3, caspase- 8 and caspase-9 activity in the miR-210 inhibitor group were significantly increased compared with those in the scrambled group (all $\mathrm{P}<0.01$ ).

MiR-210 inhibition reduces cell migration and invasion in MG63 cells. To ascertain the inhibitory effect of miR-210 inhibitor on cell motility in MG63 cells, scratch wound migration assays were performed. MG63 cells transfected with miR-210 inhibitor migrated at a significantly decreased rate compared with that of cells transfected with the negative control ( $\mathrm{P}<0.01$; Fig. 4A and $\mathrm{B})$.

In addition, the ability of miR-210 to affect the invasiveness of MG63 cells was further investigated using the Transwell assay. The results showed that miR-210 inhibition significantly inhibited cell invasion compared with that in the scrambled group $(\mathrm{P}<0.01$; Fig. $4 \mathrm{C}$ and $\mathrm{D})$.

To determine the potential mechanism of the effect of miR-210 on cell migration and invasion, the levels of the cell invasion-associated proteins VEGF, MMP-2 and MMP-9 were assessed using western blot analysis. As shown in Fig. 4E and F, VEGF, MMP-2 and MMP-9 protein expression were significantly decreased in the miR-210 inhibitor group compared with that in the scrambled group $(\mathrm{P}<0.05)$.

miR-210 inhibitor suppresses tumor growth in a nude mouse model. To further determine whether miR-210 affects OS tumor growth in vivo, MG63 cells engineered to stably overexpress miR-210 inhibitor or the scrambled sequence were subcutaneously inoculated into nude mice $(n=10)$, respectively, and the tumor growth was measured over 30 days. As shown in Fig. 5A, tumors in the miR-210 inhibitor group were significantly smaller than tumors in the negative control group. The average volume and weight of the tumors in miR-210 inhibitor group were significantly reduced compared with those in the scrambled group $(\mathrm{P}<0.05$; Fig. $5 \mathrm{~B}$ and $\mathrm{C})$. These data collectively indicated that miR-210 was able to suppress OS tumor growth in vivo.

\section{Discussion}

The results of the present study showed that miR-210 was increased in human osteosarcoma cell lines compared with that in h-OB osteoblasts cells. Furthermore, the results indicated that downregulation miR-210 inhibited cell migration and invasion of MG63 cells. In addition, downregulation miR-210 suppressed tumor growth of OS in nude mice. To the best our knowledge, the present study was the first to show that miR-210 has a crucial role in OS development and progression.

miR-210, an intronic miRNA, is located within the genomic loci of transcript AK123483 (16). miR-210 has been reported to be involved in the regulation of a hypoxia-inducible factor-1 (HIF-1)-dependent (17,18), HIF-2-dependent (17) and HIF-independent (19) pathways. miR-210 has also been identified a variety of functionally important targets involved in a wide range of physiological processes, including cell cycle regulation, differentiation, DNA damage repair, cell survival, angiogenesis as well as metabolism $(20,21)$. miR-210 has been shown to be overexpressed in a variety of malignancies, including pancreatic cancer (22), head and neck squamous cell carcinomas (23), breast cancer (24), lung cancer (25), renal cell cancer (26), non-small lung cancer (27) and osteosarcoma (14). Most studies have reported that miR-210 may act as an oncogenic miRNA and is associated with poor prognosis in a number of human epithelial cancer types (27-31). However, certain studies have indicated that miR-210 expression is lost during tumorigenesis and that the miRNA exerts a tumor-suppressor effect on human epithelial ovarian and esophageal squamous cell carcinoma $(32,33)$. These findings suggested that miR-210 may have important roles during tumorigenesis and cancer progression and may exert various effects on different cancer types. Regarding OS, previous studies demonstrated that miR-210 was upregulated in OS tissue and an OS cell line $(13,14)$. Of note, miR-210 was found to be a positive regulator of osteoblastic differentiation through the inhibition of activin A receptor type 1B (34). In line with the results of previous studies, the present study found that miR-210 was increased in human osteosarcoma cell lines, and that downregulation of miR-210 inhibited tumor growth of osteosarcoma in vitro and in vivo. The present results, together with those of other studies, suggested that miR-210 may act as an oncogenic miRNA and have crucial roles in OS tumorigenesis.

With the development of bioinformatic miRNA target prediction tools as well as the improvement of experimental approaches, numerous diverse targets of miR-210 have been identified, including E2F3, NPTX1, RAD52, ACVR1B, MNT, CASP8AP2, FGFRL1 and HOXA1 (15,35-37). It has been shown that miR-210 targets $>35$ genes $(36,37)$. The present study demonstrated that miR-210 is involved in OS cell proliferation, clonogenicity, migration and invasion, as well as cell apoptosis via targeting multiple genes. 
In conclusion, the results of the present study provided novel evidence that downregulation of miR-210 can inhibit cell proliferation, clonogenicity, migration, invasion, as well as induced G1 arrest and cell apoptosis in vitro, as well as suppress tumor growth in vivo. The findings therefore implied that miR-210 may be a potential therapeutic target for osteosarcoma.

\section{References}

1. Ottaviani G, Robert RS, Huh WW, Palla S and Jaffe N: Sociooccupational and physical outcomes more than 20 years after the diagnosis of osteosarcoma in children and adolescents: limb salvage versus amputation. Cancer 119: 3727-3736, 2013.

2. Ottaviani G and Jaffe N: The epidemiology of osteosarcoma. Cancer Treat Res 152: 3-13, 2009.

3. Yang J and Zhang W: New molecular insights into osteosarcoma targeted therapy. Curr Opin Oncol 25: 398-406, 2013.

4. Calin GA, Sevignani C, Dumitru CD, et al: Human microRNA genes are frequently located at fragile sites and genomic regions involved in cancers. Proc Natl Acad Sci USA 101: 2999-3004, 2004

5. Kim VN, Han J and Siomi MC: Biogenesis of small RNAs in animals. Nat Rev Mol Cell Biol 10: 126-139, 2009.

6. Bartel DP: MicroRNAs: target recognition and regulatory functions. Cell 136: 215-233, 2009.

7. Valencia-Sanchez MA, Liu J, Hannon GJ and Parker R: Control of translation and mRNA degradation by miRNAs and siRNAs. Genes Dev 20: 515-524, 2006.

8. Erhard F, Haas J, Lieber D, et al: Widespread context dependency of microRNA-mediated regulation. Genome Res 24: 906-919, 2014

9. Almeida MI, Reis RM and Calin GA: MicroRNA history: discovery, recent applications and next frontiers. Mutat Res 717: $1-8,2011$.

10. Kloosterman WP and Plasterk RH: The diverse functions of microRNAs in animal development and disease. Dev Cell 11: 441-450, 2006.

11. $\mathrm{Xu} \mathrm{Z}$ and Wang $\mathrm{T}$ : miR-214 promotes the proliferation and invasion of osteosarcoma cells through direct suppression of LZTS1. Biochem Biophys Res Commun 449: 190-195, 2014.

12. Li E, Zhang J, Yuan T and Ma B: miR-145 inhibits osteosarcoma cells proliferation and invasion by targeting ROCK1. Tumour Biol 35: 7645-7650, 2014

13. Lulla RR, Costa FF, Bischof JM, Chou PM, de F Bonaldo M, Vanin EF and Soares MB: Identification of differentially expressed microRNAs in osteosarcoma. Sarcoma 2011: 732690, 2011

14. Cai H, Lin L, Cai H, Tang M and Wang Z: Prognostic evaluation of microRNA-210 expression in pediatric osteosarcoma. Med Oncol 30: 499, 2013

15. Fasanaro P, Greco S, Lorenzi M, et al: An integrated approach for experimental target identification of hypoxia-induced miR-210. J Biol Chem 284: 35134-35143, 2009.

16. Giannakakis A, Sandaltzopoulos R, Greshock J, et al: miR-210 links hypoxia with cell cycle regulation and is deleted in human epithelial ovarian cancer. Cancer Biol Ther 7: 255-264, 2008

17. McCormick RI, Blick C, Ragoussis J, et al: miR-210 is a target of hypoxia-inducible factors 1 and 2 in renal cancer, regulates ISCU and correlates with good prognosis. Br J Cancer 108: 1133-1142, 2013.
18. Huang X, Ding L, Bennewith KL, Tong RT, Welford SM, Ang KK, Story M, Le QT and Giaccia AJ: Hypoxia-inducible mir-210 regulates normoxic gene expression involved in tumor initiation. Mol Cell 35: 856-867, 2009.

19. Takikawa T, Masamune A, Hamada S, Nakano E, Yoshida N and Shimosegawa T: miR-210 regulates the interaction between pancreatic cancer cells and stellate cells. Biochem Biophys Res Commun 437: 433-439, 2013

20. Devlin C, Greco S, Martelli F and Ivan M: miR-210: More than a silent player in hypoxia. IUBMB Life 63: 94-100, 2011.

21. Chan SY and Loscalzo J: MicroRNA-210: a unique and pleiotropic hypoxamir. Cell Cycle 9: 1072-1083, 2010.

22. Greither T, Grochola LF, Udelnow A, Lautenschlager C, Wurl P and Taubert H: Elevated expression of microRNAs 155, 203, 210 and 222 in pancreatic tumors is associated with poorer survival. Int J Cancer 126: 73-80, 2010.

23. Gee HE, Camps C, Buffa FM, et al: hsa-mir-210 is a marker of tumor hypoxia and a prognostic factor in head and neck cancer. Cancer 116: 2148-2158, 2010.

24. Foekens JA, Sieuwerts AM, Smid M, et al: Four miRNAs associated with aggressiveness of lymph node-negative, estrogen receptor-positive human breast cancer. Proc Natl Acad Sci USA 105: 13021-13026, 2008.

25. Puissegur MP, Mazure NM, Bertero T, et al: miR-210 is overexpressed in late stages of lung cancer and mediates mitochondrial alterations associated with modulation of HIF-1 activity. Cell Death Differ 18: 465-478, 2011.

26. Juan D, Alexe G, Antes T, et al: Identification of a microRNA panel for clear-cell kidney cancer. Urology 75: 835-841, 2010.

27. Eilertsen M, Andersen S, Al-Saad S, et al: Positive prognostic impact of miR-210 in non-small cell lung cancer. Lung Cancer 83: 272-278, 2014

28. Ying Q, Liang L, Guo W, et al: Hypoxia-inducible microRNA-210 augments the metastatic potential of tumor cells by targeting vacuole membrane protein 1 in hepatocellular carcinoma. Hepatology 54: 2064-2075, 2011.

29. Hong L, Yang J, Han Y, Lu Q, Cao J and Syed L: High expression of miR-210 predicts poor survival in patients with breast cancer: a meta-analysis. Gene 507: 135-138, 2012.

30. Wotschofsky Z, Busch J, Jung M, et al: Diagnostic and prognostic potential of differentially expressed miRNAs between metastatic and non-metastatic renal cell carcinoma at the time of nephrectomy. Clin Chim Acta 416: 5-10, 2013.

31. Qu A, Du L, Yang Y, et al: Hypoxia-inducible MiR-210 is an independent prognostic factor and contributes to metastasis in colorectal cancer. PLoS One 9: e90952, 2014.

32. Giannakakis A, Sandaltzopoulos R, Greshock J, et al: miR-210 links hypoxia with cell cycle regulation and is deleted in human epithelial ovarian cancer. Cancer Biol Ther 7: 255-264, 2008.

33. Tsuchiya S: The role of microRNA-210 in esophageal squamous cell carcinoma. Yakugaku Zasshi 132: 1069-1073, 2012 (In Japanese).

34. Mizuno Y, Tokuzawa Y, Ninomiya Y, et al: miR-210 promotes osteoblastic differentiation through inhibition of AcvRlb. FEBS Lett 583: 2263-2268, 2009.

35. Chan YC, Banerjee J, Choi SY and Sen CK: miR-210: the master hypoxamir. Microcirculation 19: 215-223, 2012.

36. Mei Y, Gao C, Wang K, et al: Effect of microRNA-210 on prognosis and response to chemotherapeutic drugs in pediatric acute lymphoblastic leukemia. Cancer Sci 105: 463-472, 2014.

37. Qin Q, Furong W and Baosheng L: Multiple functions of hypoxia-regulated miR-210 in cancer. J Exp Clin Cancer Res 33: 50, 2014. 